\title{
Gut barrier disruption by an enteric bacterial pathogen accelerates insulitis in NOD mice
}

\author{
A. S. Lee • D. L. Gibson • Y. Zhang • H. P. Sham • \\ B. A. Vallance $\cdot$ J. P. Dutz
}

Received: 23 August 2009 / Accepted: 6 November 2009 /Published online: 13 December 2009

(C) Springer-Verlag 2009

\begin{abstract}
Aims/hypothesis Increased exposure to enteric microbes as a result of intestinal barrier disruption is thought to contribute to the development of several intestinal inflammatory diseases; however, it less clear whether such exposure modulates the development of extra-intestinal inflammatory and autoimmune diseases. The goal of this study was to examine the potential role of pathogenic enteric microbes and intestinal barrier dysfunction in the pathogenesis of type 1 diabetes.

Methods Using NOD mice, we assessed: (1) intrinsic barrier function in mice at different ages by measuring serum levels of FITC-labelled dextran; and (2) the impact on insulitis development of infection by strains of an enteric bacterial pathogen (Citrobacter rodentium) either capable (wild-type) or incapable (lacking Escherichia coli secreted
\end{abstract}

A. S. Lee and D. L. Gibson are joint first authors; B. A. Vallance and J. P. Dutz are joint senior authors.

A. S. Lee $\cdot$ Y. Zhang $\cdot$ J. P. Dutz

Department of Medicine, University of British Columbia,

Vancouver, BC, Canada

A. S. Lee $\cdot$ Y. Zhang $\cdot$ J. P. Dutz $(\bowtie)$

Department of Dermatology and Skin Science,

University of British Columbia,

835 West 10th Ave,

Vancouver, BC, Canada V5Z 4E8

e-mail: dutz@interchange.ubc.ca

D. L. Gibson $\cdot$ H. P. Sham • B. A. Vallance $(\square)$

Department of Paediatrics, Division of Gastroenterology,

University of British Columbia,

British Columbia Children's Hospital,

Room K4-188, 4480 Oak Street, Ambulatory Care Building,

Vancouver, BC, Canada V6H 3V4

e-mail: bvallance@cw.bc.ca protein $\mathrm{F}$ virulence factor owing to deletion of the gene $[\Delta e s p F])$ of causing intestinal epithelial barrier disruption. Results Here we demonstrate that prediabetic (12-weekold) NOD mice display increased intestinal permeability compared with non-obese diabetes-resistant and C57BL/6 mice. We also found that young (4-week-old) NOD mice infected with wild-type $C$. rodentium exhibited accelerated development of insulitis in concert with infection-induced barrier disruption. In contrast, insulitis development was not altered in NOD mice infected with the non-barrier-disrupting $\Delta e s p F$ strain. Moreover, C. rodentium-infected NOD mice demonstrated increased activation and proliferation of pancreatic-draining lymph node $\mathrm{T}$ cells, including diabetogenic $\mathrm{CD} 8^{+} \mathrm{T}$ cells, compared with uninfected NOD mice. Conclusions/interpretation This is the first demonstration that a loss of intestinal barrier integrity caused by an enteric bacterial pathogen results in the activation of diabetogenic $\mathrm{CD}^{+} \mathrm{T}$ cells and modulates insulitis.

Keywords Citrobacter rodentium - $\Delta e s p F$ Citrobacter rodentium - Enteric bacterial infection - Gut barrier disruption · Insulitis · Intestinal barrier · NOD mice · Type 1 diabetes

$\begin{array}{ll}\text { Abbreviations } \\ \Delta e s p F & \begin{array}{l}\text { Strain lacking Escherichia coli } \text { secreted protein F } \\ \text { virulence factor }\end{array} \\ \text { CFSE } & \text { Carboxyfluorescein succinimidyl ester } \\ \text { CFU } & \text { Colony-forming unit } \\ \text { CTL } & \text { Cytotoxic T lymphocyte } \\ \text { espF } & \text { Escherichia coli secreted protein F virulence factor } \\ \text { GI } & \text { Gastrointestinal } \\ \text { IGRP } & \begin{array}{l}\text { Islet-specific glucose-6-phosphatase catalytic } \\ \text { subunit-related protein }\end{array} \\ \text { LN } & \text { Lymph node }\end{array}$




$\begin{array}{ll}\text { NOR } & \text { Non-obese diabetes-resistant } \\ \text { TLR } & \text { Toll-like receptor } \\ \text { WT } & \text { Wild-type }\end{array}$

\section{Introduction}

Normally, the intestinal epithelium provides a protective barrier preventing both pathogenic and commensal bacteria from escaping from the intestinal lumen and activating the systemic immune system. Disruption of this delicate barrier promotes the initiation and development of intestinal autoimmune diseases such as coeliac disease [1] and inflammatory bowel disease [2]. Impaired intestinal barrier function has also been detected in rodent models of type 1 diabetes [3-7] and in type 1 diabetes patients and their relatives [8-12]. Changes in the intestine, induced by dietary or enteric antigens, have been proposed as causative factors in the course of type 1 diabetes $[13,14]$; however, the role of the gut and enteric microbes in type 1 diabetes remains unclear. We thus propose that impaired intestinal barrier function in diabetes-prone individuals may allow commensal or pathogenic enteric bacterial antigens to elicit inflammatory signals that promote type 1 diabetes.

Type 1 diabetes is the result of an immune-mediated destruction of insulin-producing beta cells in the pancreas, predominantly by cytotoxic T lymphocytes (CTLs) [15]. The primary activation of diabetogenic CTLs occurs within the pancreatic and gut-associated lymph nodes (LNs) $[16,17]$. Lymphocytes within the pancreatic LNs thus communicate with the intestinal immune system, and the gut and its luminal contents may contribute to the initiation of type 1 diabetes [18]. Luminal antigens from the gut are preferentially transported to the pancreatic LNs, resulting in the local proliferation of antigen-specific CTLs [18]. Additionally, diabetogenic CTLs express the gut-homing receptor $\alpha 4 \beta 7$ integrin [19-21] and CTLs activated in the gastrointestinal (GI) tract of NOD mice home to islets that express the mucosal-homing receptor mucosal addressin cell adhesion molecule-1 [19-21].

Based on these findings, we hypothesised that an impaired intestinal barrier function exposing the immune system to GI microbes and other luminal antigens might provide an inflammatory stimulus that drives the autoimmune response in type 1 diabetes. In this study, we demonstrate that prediabetic NOD mice develop an intrinsic intestinal barrier dysfunction by 12 weeks of age. Moreover, colonic infection of young (4-week-old) NOD mice by a bacterial pathogen (wild-type [WT] Citrobacter rodentium) that specifically damages the intestinal epithelial barrier promotes the development of insulitis, which was not observed when these mice were infected with a mutant strain of $C$. rodentium (lacking Escherichia coli secreted protein $\mathrm{F}$ virulence factor $[\Delta e s p F]$ ) that is unable to disrupt the intestinal epithelial barrier [22]. We show that enteric infections can modify islet inflammation in type 1 diabetes and demonstrate that enteric infection-induced damage of intestinal barrier function may affect the early stages of development of type 1 diabetes.

\section{Methods}

Mice, bacteria and infection 8.3 NOD mice, expressing the rearranged $\mathrm{T}$ cell receptor genes of the diabetogenic CTL clone NY8.3, have been previously described [23] and were provided by P. Santamaria (University of Calgary, Calgary, AB, Canada). 8.3 NOD mice and NOD mice (Jackson Laboratory, Bar Harbor, ME, USA) were housed and bred under SPF conditions at the Child and Family Research Institute (Vancouver, BC, Canada), and used between 4 and 12 weeks of age. Mice were fed with LabDiet 5053 Irradiated Picolab Rodent Diet 20 (PMI Nutrition International, Jamieson's Pet Foods International, Vancouver, BC, Canada). Infection of mice and culturing of WT and $\Delta e s p F$ C. rodentium was performed as previously described [22, 24]. In brief, mice were infected by oral gavage with $0.1 \mathrm{ml}$ of an overnight bacterial culture in Luria broth containing approximately $2.5 \times 10^{8}$ colony-forming units (CFUs) of WT or $\Delta e s p F C$. rodentium. To enumerate $C$. rodentium, whole gut tissues (including stools) or LNs were collected from infected mice, homogenised, plated on MacConkey plates, incubated at $37^{\circ} \mathrm{C}$, and colonies counted the following day [22, 24]. Experiments were conducted as approved by the institutional animal ethics committee.

FITC-dextran assay The FITC-dextran assay was performed as described [24]. Briefly, for assessment of barrier function in the upper GI tract, $0.15 \mathrm{ml}$ of $80 \mathrm{~g} / 1$ of FITCdextran (FD4; Sigma-Aldrich, Oakville, ON, Canada) in PBS was introduced to mice by oral gavage, whereas $0.1 \mathrm{ml}$ was introduced by enema for studies assessing colonic barrier function following $C$. rodentium infection. After $2 \mathrm{~h}$ (enema) or $4 \mathrm{~h}$ (oral gavage), serum was collected by cardiac puncture and measured with a fluorimeter (Victor X3; PerkinElmer, Waltham, MA, USA).

Insulitis scoring and diabetes assessment Paraffin-embedded sections of the pancreas were stained with haematoxylin and eosin, and examined by light microscopy (BX61TRF; Olympus, Tokyo, Japan). Insulitis severity was assessed as described [25] in normoglycaemic mice by an observer blinded to tissue source by counting at least 30 pancreatic islets per mouse from four parallel sections of different cut levels of the pancreas. Mice that became diabetic within 
12 weeks of age were censored in each group (3/30 uninfected; $2 / 25$ WT $C$. rodentium-infected; and $4 / 24$ $\triangle e s p F C$. rodentium-infected NOD mice) because of a paucity of islets available for counting. Blood glucose was measured using Elite glucostrips and glucometer (Bayer, Etobicoke, ON, Canada). Animals were considered to be diabetic when two consecutive measurements exceeded $14 \mathrm{mmol} / \mathrm{l}$. The degree of insulitis was classified into four categories: 0 , no insulitis; 1 , peri-insulitis with or without minimal lymphocytic infiltration in islets; 2 , invasive insulitis with $<50 \%$ of lymphocytic infiltration of islets; or 3 , invasive insulitis with $\geq 50 \%$ of lymphocytic infiltration of islets.

Adoptive transfer, LN preparation and flow cytometry analysis Naive $8.3 \mathrm{NOD}_{\mathrm{CD}}{ }^{+} \mathrm{T}$ cells (islet-specific glucose-6-phosphatase catalytic subunit-related protein [IGRP]-specific [islet antigen] $\mathrm{CD} 8^{+} \mathrm{T}$ cells) were prepared and purified from the peripheral LNs and spleens of transgenic mice and labelled with CFSE (carboxyfluorescein succinimidyl ester $5 \mu \mathrm{mol} / \mathrm{l}$; Molecular Probes-Invitrogen, Burlington, ON, Canada) [26]. The labelled cells $\left(1 \times 10^{7}\right)$ were transferred to WT $C$. rodentium-infected NOD recipients i.v. via a tail vein at 10 days after infection. $\mathrm{LN}$ single-cell suspensions were prepared, stained and examined on a FACSCalibur flow cytometer (BD Bioscience, San Jose, CA, USA) as described [26].

Statistical analysis Two-tailed Student's $t$ test and nonparametric Mann-Whitney $t$ tests were used to calculate statistical significance where indicated. All statistical analysis was conducted using Prism 3 GraphPad software (La Jolla, CA, USA).

\section{Results}

Intrinsic intestinal barrier dysfunction in prediabetic NOD mice Recent evidence suggests a link between intestinal barrier dysfunction, subsequent enteropathies and an increased risk of type 1 diabetes in both rodent models and humans $[1,2,4-6,11,12,14]$. To explore the role of intestinal barrier permeability in the pathogenesis of type 1 diabetes, we examined intestinal epithelial barrier function in the NOD mouse.

Prediabetic (12-week-old) NOD mice exhibited increased serum levels of FITC-dextran compared with agematched C57BL/6 and non-obese diabetes-resistant (NOR) mice following oral gavage (Fig. 1). However, younger prediabetic (4 and 8 weeks) NOD mice did not exhibit differences in serum FITC-dextran levels compared with age-matched C57BL/6 and NOR mice (data not shown). The increased barrier permeability occurs at the time of insulitis and precedes the development of type 1 diabetes.

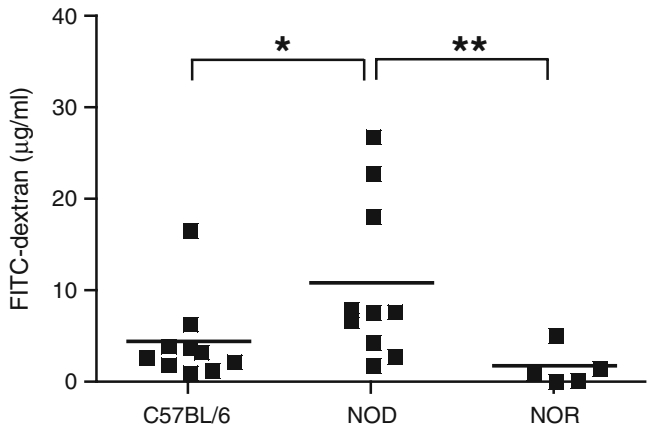

Fig. 1 Intrinsic intestinal barrier dysfunction in prediabetic NOD mice. Assessment of the intestinal barrier integrity with orally administered FITC-dextran in 12-week-old C57BL/6 $(n=10)$, NOD $(n=10)$ and NOR $(n=5)$ mice. Points represent the serum FITCdextran levels of individual mice. Data are representative of two independent experiments. ${ }^{*} p<0.05 ;{ }^{* *} p<0.005$ by non-parametric Mann-Whitney $t$ test

WT C. rodentium infection disrupts intestinal barrier function in NOD mice To determine if intestinal barrier disruption affects diabetogenic autoimmune inflammation, we determined if disruption of barrier integrity at a young age (4 weeks) in NOD mice would alter insulitis. WT $C$. rodentium, an enteric bacterial pathogen that disrupts the intestinal epithelium, was used to infect young prediabetic (4-week-old) NOD mice. WT $C$. rodentium is a gramnegative attaching/effacing mucosal pathogen that rapidly infects the colonic epithelium of mice in an identical fashion to that used by enteropathogenic E. coli [27]. WT C. rodentium-infected mice develop a colitis characterised by inflammatory cell infiltration, crypt cell hyperplasia, goblet cell depletion and significant intestinal barrier disruption that are mediated primarily by the virulence factor espF $[22,27]$. WT C. rodentium infection increased intestinal barrier permeability in NOD mice as measured by levels of FITC-dextran recovered from the serum of infected mice following rectal instillation (Fig. 2a). A similar observation was observed in C57BL/6 mice infected with WT C. rodentium (data not shown).

C. rodentium translocates to the mesenteric and pancreatic $L N s$ of infected NOD mice We next assessed WT $C$. rodentium CFUs within the colon and caecum, and determined if the resulting barrier disruption led to this pathogen reaching the mesenteric and pancreatic LNs. WT C. rodentium counts were higher in these $\mathrm{LNs}$, and in the caecum and colon of infected NOD mice compared with infected C57BL/6 mice (Fig. 2b). There were no differences in WT $C$. rodentium numbers detected in the pancreas, spleen or liver in either mouse strain (data not shown). The WT $C$. rodentium recovered from the LNs of infected mice may reflect those microbes specifically transported to these lymphoid tissues by dendritic cells or macrophages, either as a result of sampling the intestinal lumen or following 
a
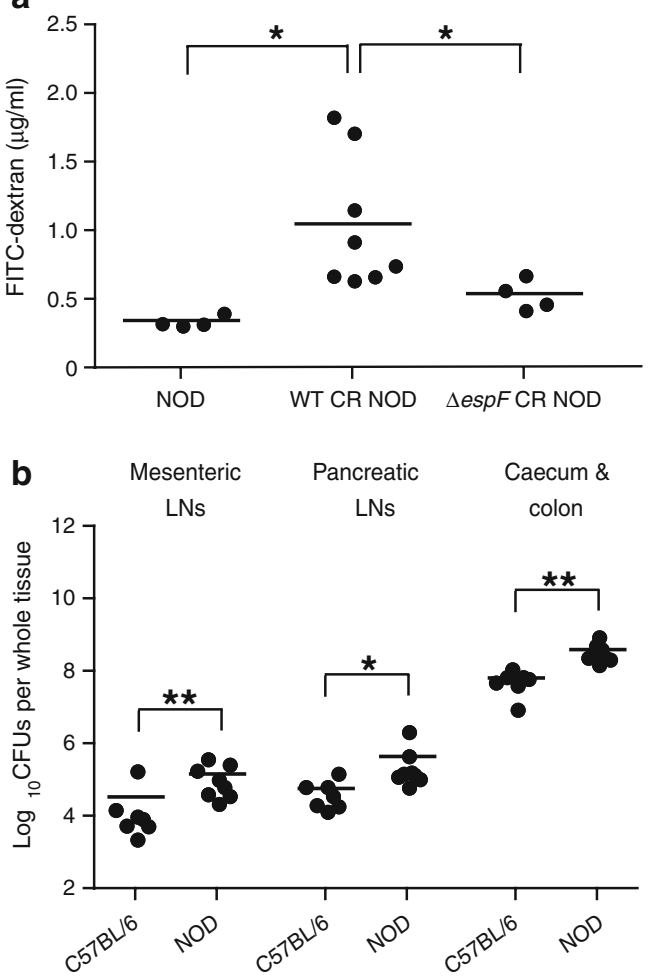

Fig. 2 WT but not $\triangle \operatorname{espF} C$. rodentium infection increases intestinal barrier dysfunction and WT $C$. rodentium increases translocation to the mesenteric and pancreatic LNs in NOD mice. a Assessment of the intestinal barrier integrity with enema-administered FITCdextran in infected (WT or $\triangle \operatorname{espF} C$. rodentium [CR]) vs noninfected 4-week-old NOD mice at day 10 after infection. b Increased WT $C$. rodentium CFUs were found in the mesenteric and pancreatic LNs and the caecum and colon of infected NOD vs infected C57BL/6 mice at day 8 after infection. There were no WT C. rodentium CFUs found in non-infected mice. Data are representative of two independent experiments. ${ }^{*} p<0.05 ;{ }^{*} p<0.009$ by non-parametric Mann-Whitney $t$ test

phagocytosis of $C$. rodentium that passively leaked across a disrupted intestinal barrier.

WT but not $\triangle \operatorname{espF~} C$. rodentium accelerates insulitis in NOD mice WT $C$. rodentium infection of young (4-weekold) prediabetic NOD mice accelerated the development of insulitis as assessed at 12 weeks of age ( 8 weeks after infection). Infection significantly increased the percentage of islets exhibiting $\geq 50 \%$ lymphocytic infiltration compared with age-matched controls (Fig. 3a, e). Correspondingly, there were significantly fewer islets in these mice exhibiting no infiltration (Fig. 3b).

To determine if the accelerated insulitis was because of $C$. rodentium's ability to disrupt the intestinal epithelial barrier integrity, we also infected NOD mice with a mutant strain of $C$. rodentium that lacks espF $(\Delta e s p F)$. This bacterial protein plays a primary role in $C$. rodentium's ability to disrupt intestinal epithelial barrier function [22].
Infection of NOD mice with $\Delta e s p F C$. rodentium did not increase intestinal barrier permeability as measured by serum FITC-dextran levels (Fig. 2a), nor did it increase insulitis scores (Fig. 3), compared with uninfected mice. These results indicate that intestinal barrier disruption is a prerequisite for $C$. rodentium infection to influence the development of insulitis.

WT C. rodentium increases the proliferation and activation of $C D 8^{+}$and $C D 4^{+} T$ cells in NOD mice The increase in lymphocytic infiltration into the islets in WT $C$. rodentiuminfected NOD mice suggests an increase in the priming of diabetogenic CTLs. 8.3 NOD $\mathrm{CD}^{+} \mathrm{T}$ cells recognise an immuno-dominant peptide from IGRP, termed IGRP ${ }_{206-214}$, a protein expressed by pancreatic beta cells, and these cells are representative of a significant fraction of $\mathrm{CD} 8^{+} \mathrm{T}$ cells in pancreatic islets at the onset of inflammation [23, 28, 29]. Adoptive transfer of IGRP-specific (islet antigen) $\mathrm{CD} 8^{+} \mathrm{T}$ cells [26] into infected NOD mice confirmed an increase in the proliferation and activation of these cells in the pancreatic LNs (Fig. 4a). In addition, infected NOD mice displayed increased expression of the early and late activation markers, CD69 and CD25, respectively, on polyclonal $\mathrm{CD}^{+}$and $\mathrm{CD} 4^{+} \mathrm{T}$ cells in the mesenteric and pancreatic LNs (Fig. 4c-e). Thus, WT C. rodentium infection enhances the activation of polyclonal and diabetogenic CTLs in the early stages of type 1 diabetes.

\section{Discussion}

The present study demonstrates that alterations in GI barrier function modify insulitis, a precursor stage of the extraintestinal autoimmune disease, type 1 diabetes. NOD mice develop an overt and spontaneous intestinal barrier defect at the time of insulitis that precedes the development of type 1 diabetes. Variability in the levels of spontaneous barrier disruption suggests a heterogeneous defect. Importantly, infection of NOD mice with the enteric bacterial pathogen, $C$. rodentium, accelerates the development of insulitis, but only when this pathogen is capable of disrupting colonic epithelial barrier function. The increased lymphocytic infiltration within the islets of WT $C$. rodentium-infected NOD mice occurs concurrently with the increased activation of both polyclonal and diabetogenic CTLs. These data suggest that exposure to enteric pathogens (i.e. bacteria as demonstrated here, or viral pathogens) and possibly other dietary or gut antigens may increase the risk of activating diabetogenic CTLs and subsequently developing insulitis and type 1 diabetes.

There is mounting evidence suggesting that intrinsic or induced intestinal barrier defects skew the intestinal immune environment or the commensal microbiota to promote type 1 diabetes [14]. For example, type 1 diabetes 
a
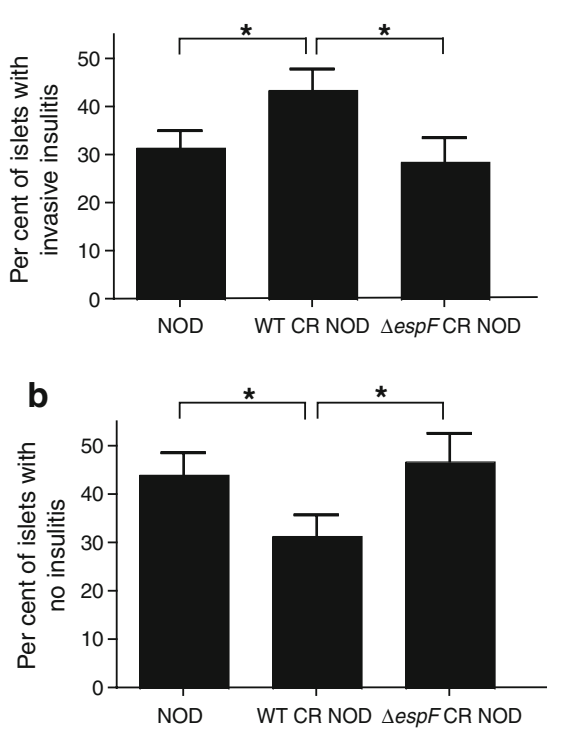

C

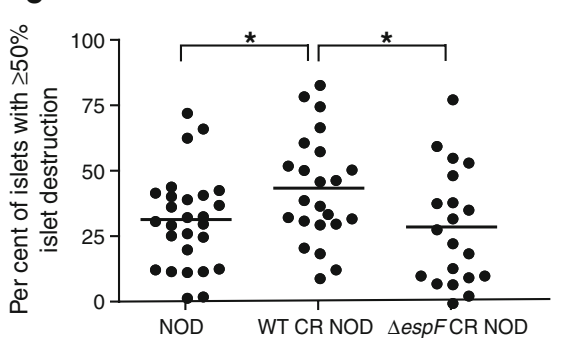

d

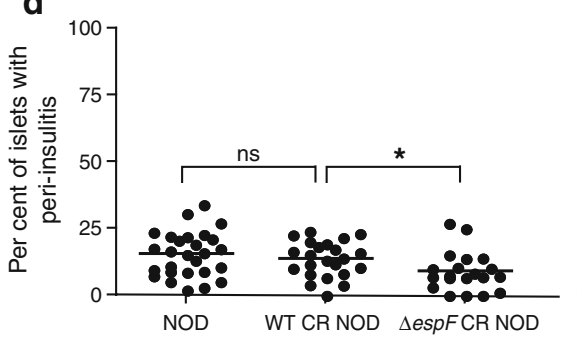

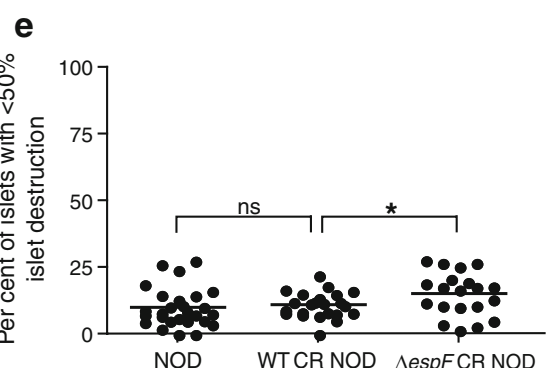

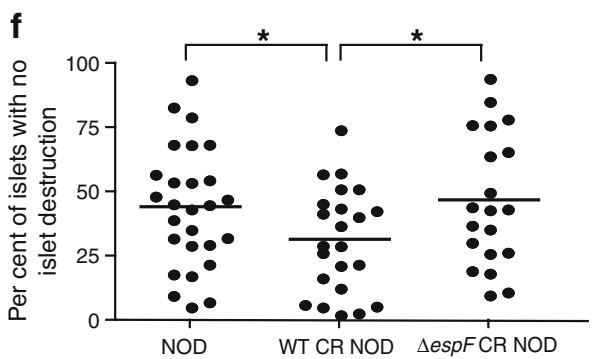

Fig. 3 a, b WT but not $\triangle e s p F C$. rodentium (CR) accelerates the development of insulitis in NOD mice. a Per cent of islets with invasive insulitis ( $\geq 50 \%$ lymphocytic infiltration in islets) (score 3 ). $\mathbf{b}$ Per cent of islets with no insulitis (score 0 ). c-f Individual insulitis score of the pancreas in either WT or $\triangle \operatorname{espF} \mathrm{CR}$-infected and noninfected NOD mice at 12 weeks of age. c Score 3, invasive insulitis with $\geq 50 \%$ of lymphocytic infiltration of islets. d Score 1, peri- insulitis with or without minimal lymphocytic infiltration in islets. e Score 2 , invasive insulitis with $<50 \%$ of lymphocytic infiltration of islets. f Score 0 , no insulitis. Data are representative of three independent experiments in non-infected NOD mice $(n=27)$ or WT CR-infected $(n=23)$ or $\Delta e s p F$ CR-infected $(n=20)$ NOD mice. ${ }^{*} p<$ 0.05 by non-parametric Mann-Whitney $t$ tests; ns: not significant

\section{a}

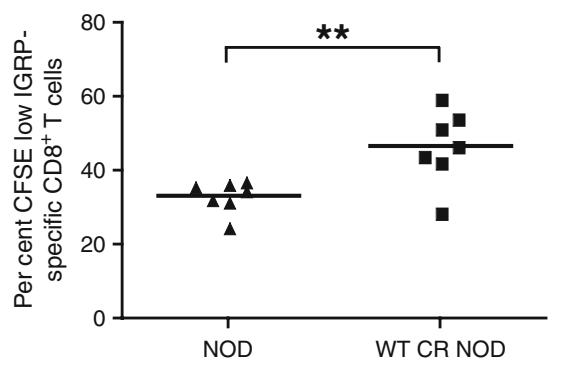

b

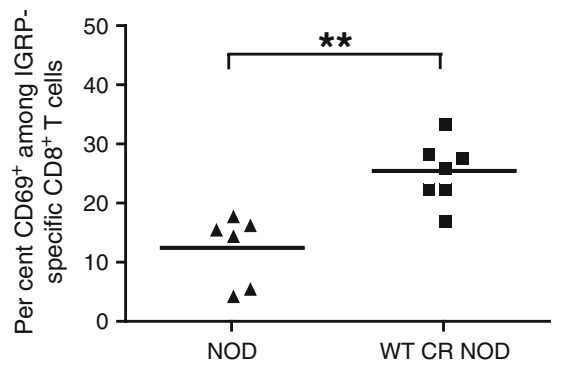

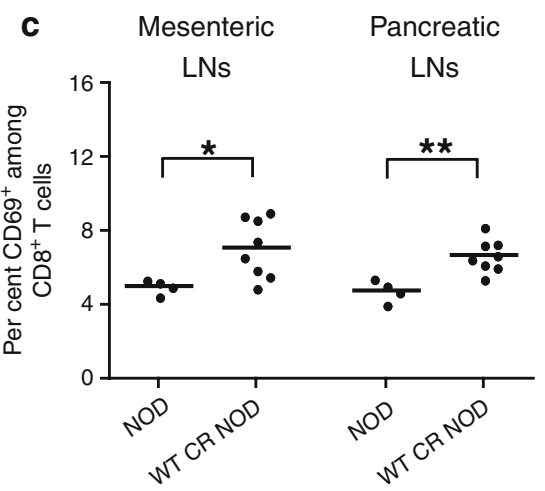

Fig. 4 WT $C$. rodentium (CR) increases the proliferation and activation of diabetogenic $\mathrm{CD} 8^{+} \mathrm{T}$ cells and the activation of polyclonal $\mathrm{CD} 8^{+}$and $\mathrm{CD} 4^{+} \mathrm{T}$ cells. a, b Increased proliferation and activation of IGRP-specific $\mathrm{CD}^{+} \mathrm{T}$ cells in the pancreatic $\mathrm{LNs}$ of infected NOD mice. c-e Increased upregulation of CD69 or CD25 in
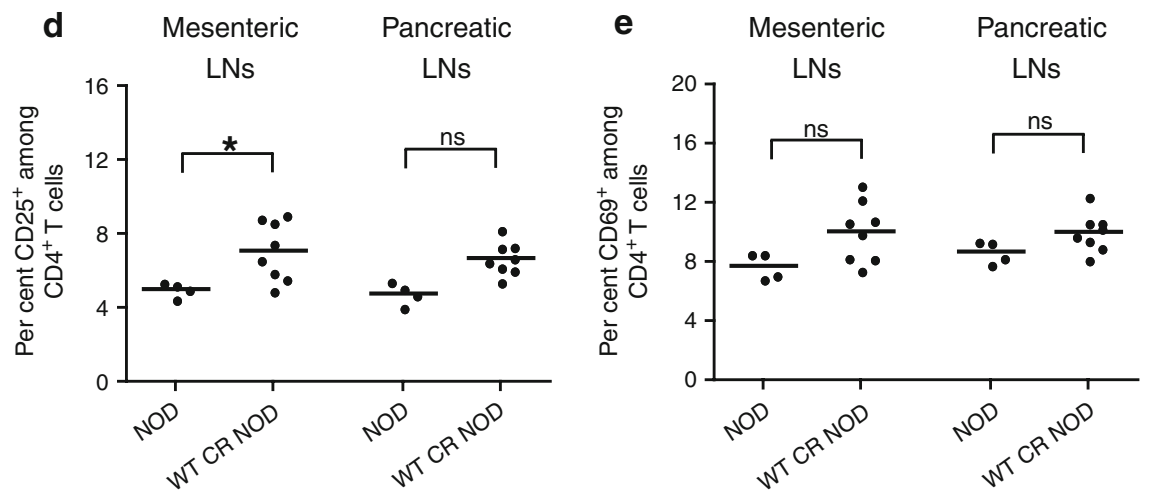

polyclonal $\mathrm{CD}^{+}$and $\mathrm{CD}^{+} \mathrm{T}$ cells in the mesenteric or pancreatic LNs of infected NOD mice. Data are representative of two independent experiments. ${ }^{*} p<0.05 ;{ }^{*} p<0.009$ by unpaired $t$ tests; ns: not significant 
has been epidemiologically linked to coeliac disease, where gut barrier dysfunction has also been proposed as a predisposing factor [2, 13]. Wheat gluten has been identified as a main contributor of gut barrier dysfunction in coeliac disease and as a potential source of gut barrier dysfunction in type 1 diabetes [2,13]. NOD mice fed a wheat gluten diet develop a Th1 cytokine bias in the gut [30]. This diet promotes small intestinal enteropathy [31], and increases the incidence of diabetes [31]. However, a recent report has shown conflicting evidence of the role of wheat gluten in type 1 diabetes in NOD mice: NOD mice fed either gluten-enriched or gluten-free diets showed a delay in the development of type 1 diabetes [32]. Dietary antigens inclusive of wheat gluten may thus have an important role in modulating diabetes onset. Additionally, viral infections are associated with the onset of type 1 diabetes (particularly enteroviruses and rotaviruses) and also affect gut permeability [33, 34]. Reports have shown that children with type 1 diabetes have increased barrier defects in their small intestines ([8] and reviewed in [35]) and have a higher incidence of enteroviral infections [36]. Moreover, recent studies have also implicated innate recognition of enteric commensal microbes in the modulation of diabetes suffered by NOD mice [37]. An altered commensal gut flora because of interrupted toll-like receptor (TLR) signalling was shown to prevent diabetes. In contrast, relatively little is known about the potential role of enteric bacterial pathogens in modulating the occurrence of type 1 diabetes.

We show that prediabetic NOD mice develop an intrinsic 'leaky' gut. This finding has been well characterised in the BioBreeding rat model of type 1 diabetes $[3,5,6]$. Watts et al. [7] have suggested that intrinsic gut leakiness in BioBreeding rats may be caused by an increase in endogenous zonulin, a protein involved in modulating intestinal tight junctions. This increased zonulin has been shown to be present in type 1 diabetes patients and their relatives [11]. In contrast, the mechanism that leads to intrinsic gut leakiness in the NOD mouse remains unclear. Hadjiyanni et al. [4] described a barrier defect in the jejunum (small intestine) of 7- to 10-week-old NOD mice, but did not determine whether this defect contributes to, or alternatively is the result of islet inflammation. We demonstrated increased permeability to oral FITC-dextran (probably caused by small bowel barrier change) at 12 weeks of age (prior to the onset of diabetes), but not at 4-8 weeks. In contrast to Hadjiyanni et al., we demonstrate that infection with WT $C$. rodentium several weeks prior to the development of insulitis induces significant barrier disruption in the large intestine (using the FITC-dextran enema technique [Fig. 2a]) and accelerates insulitis (Fig. 3) in NOD mice. This acceleration was not observed when NOD mice were infected with the mutant $C$. rodentium strain $(\Delta e s p F)$ (Fig. 3) that does not damage intestinal barrier integrity
[22]. Thus, barrier dysfunction at different sites within the GI tract (small bowel or large bowel), and at different times, may modulate the development of type 1 diabetes.

While $C$. rodentium infection of young NOD mice promotes insulitis, i.v. infection with attenuated Salmonella enteriditis serotype typhimurium (Salmonella typhimurium) halted type 1 diabetes onset in NOD mice [38]. These differences in the impact on type 1 diabetes development may reflect that intestinal barrier disruption (as seen with $C$. rodentium infection) does not occur when Salmonella typhimurium is administered parenterally and suggests that disruption of the gut barrier contributes to pathogenaccelerated insulitis. This hypothesis is supported by the observation that chemical disruption of the intestine with $2 \%(\mathrm{wt} / \mathrm{vol}$.) dextran sodium sulphate in the drinking water also leads to the proliferation of diabetogenic $\mathrm{T}$ cells and accelerates development of insulitis [18].

Innate and adaptive immune responses coordinated by epithelial TLR signalling modulate the enteric flora and in turn are affected by gut microbes [37, 39-41]. Epithelial TLR signalling is critical in maintaining intestinal epithelial integrity [40]. Disruption of this homeostasis as a result of a dysregulated interaction between TLRs and enteric bacteria promotes both inflammatory bowel disease and type 1 diabetes [14, 42]. It is possible that enhanced antigen presenting cell-mediated TLR signalling is induced by enteric organisms breaching a gut barrier and that this promotes insulitis. Whether disease onset is modulated via epithelial and/or antigen presenting cell-mediated TLR or other innate signalling induced by enteric organisms (either commensals or pathogens) remains to be clarified.

Although there are no definitive clinical studies linking enteric bacterial infections to the development of human type 1 diabetes, there are a growing number of reports of type 1 diabetes patients who concomitantly present with intestinal enteropathies [8-12, 35]. Our results demonstrate that intestinal barrier dysfunction, in this case mediated by the enteric bacterial pathogen, $C$. rodentium, is a catalyst for the accelerated development of insulitis and for the priming of $\mathrm{CD} 8^{+} \mathrm{T}$ cells that are potentially diabetogenic. As enteric infections may also modulate regulatory $\mathrm{T}$ cell subsets, future studies are needed to address the ultimate impact of $C$. rodentium upon type 1 diabetes. Further studies are also warranted to determine if other enteric infections (i.e. enteroviruses) and drugs (i.e. non-steroidal anti-inflammatory drugs) that damage the intestinal barrier accelerate the development of spontaneous diabetes in diabetes-prone animal models. Conversely, manipulation of intestinal barrier integrity may offer therapeutic avenues for the treatment of type 1 diabetes.

Acknowledgements This work was supported by a grant from the Juvenile Diabetes Research Foundation to J. P. Dutz, B. A. Vallance 
and D. L. Gibson. A. S. Lee is supported by a Michael Smith Foundation of Health Research (MSFHR) Junior Graduate Studentship. D. L. Gibson is supported by a Canadian Association of Gastroenterology/AstraZeneca/CIHR Post-Doctoral Research Initiative award and an MSFHR Post-Doctoral Fellow award. B. A. Vallance is the Children with Intestinal and Liver Disorders (CHILD) Foundation Research Scholar, an MSHFR Scholar and the Canada Research Chair in Paediatric Gastroenterology. J. P. Dutz is an MSFHR Senior Scholar, Child and Family Research Institute (CFRI) Senior Scholar and Senior Scientist at the CFRI. We are grateful to the Dutz and Vallance laboratories for their comments and support.

Duality of interest The authors declare that there is no duality of interest associated with this manuscript.

\section{References}

1. Vogelsang H, Schwarzenhofer M, Oberhuber G (1998) Changes in gastrointestinal permeability in celiac disease. Dig Dis 16:333-336

2. Liu Z, Li N, Neu J (2005) Tight junctions, leaky intestines, and pediatric diseases. Acta Paediatr 94:386-393

3. Graham S, Courtois P, Malaisse WJ, Rozing J, Scott FW, Mowat AM (2004) Enteropathy precedes type 1 diabetes in the BB rat. Gut 53:1437-1444

4. Hadjiyanni I, Li KK, Drucker DJ (2009) Glucagon-like peptide-2 reduces intestinal permeability but does not modify the onset of type 1 diabetes in the nonobese diabetic mouse. Endocrinology 150:592-599

5. Meddings JB, Jarand J, Urbanski SJ, Hardin J, Gall DG (1999) Increased gastrointestinal permeability is an early lesion in the spontaneously diabetic BB rat. Am J Physiol 276:G951-G957

6. Neu J, Reverte CM, Mackey AD et al (2005) Changes in intestinal morphology and permeability in the biobreeding rat before the onset of type 1 diabetes. J Pediatr Gastroenterol Nutr 40:589-595

7. Watts T, Berti I, Sapone A et al (2005) Role of the intestinal tight junction modulator zonulin in the pathogenesis of type I diabetes in BB diabetic-prone rats. Proc Natl Acad Sci USA 102:2916-2921

8. Bosi E, Molteni L, Radaelli MG et al (2006) Increased intestinal permeability precedes clinical onset of type 1 diabetes. Diabetologia 49:2824-2827

9. Damci T, Nuhoglu I, Devranoglu G, Osar Z, Demir M, Ilkova H (2003) Increased intestinal permeability as a cause of fluctuating postprandial blood glucose levels in type 1 diabetic patients. Eur J Clin Invest 33:397-401

10. Kuitunen M, Saukkonen T, Ilonen J, Akerblom HK, Savilahti E (2002) Intestinal permeability to mannitol and lactulose in children with type 1 diabetes with the HLA-DQB $1 * 02$ allele. Autoimmunity 35:365-368

11. Sapone A, de Magistris L, Pietzak M et al (2006) Zonulin upregulation is associated with increased gut permeability in subjects with type 1 diabetes and their relatives. Diabetes 55:1443-1449

12. Secondulfo M, Iafusco D, Carratu R et al (2004) Ultrastructural mucosal alterations and increased intestinal permeability in nonceliac, type I diabetic patients. Dig Liver Dis 36:35-45

13. Lefebvre DE, Powell KL, Strom A, Scott FW (2006) Dietary proteins as environmental modifiers of type 1 diabetes mellitus. Annu Rev Nutr 26:175-202

14. Vaarala O, Atkinson MA, Neu J (2008) The 'perfect storm' for type 1 diabetes: the complex interplay between intestinal microbiota, gut permeability, and mucosal immunity. Diabetes 57:2555-2562

15. Anderson MS, Bluestone JA (2005) The NOD mouse: a model of immune dysregulation. Annu Rev Immunol 23:447-485
16. Gagnerault MC, Luan JJ, Lotton C, Lepault F (2002) Pancreatic lymph nodes are required for priming of beta cell reactive $\mathrm{T}$ cells in NOD mice. J Exp Med 196:369-377

17. Jaakkola I, Jalkanen S, Hanninen A (2003) Diabetogenic T cells are primed both in pancreatic and gut-associated lymph nodes in NOD mice. Eur J Immunol 33:3255-3264

18. Turley SJ, Lee JW, Dutton-Swain N, Mathis D, Benoist C (2005) Endocrine self and gut non-self intersect in the pancreatic lymph nodes. Proc Natl Acad Sci USA 102:17729-17733

19. Hanninen A, Salmi M, Simell O, Jalkanen S (1996) Mucosaassociated (beta 7-integrin high) lymphocytes accumulate early in the pancreas of NOD mice and show aberrant recirculation behavior. Diabetes 45:1173-1180

20. Yang XD, Michie SA, Tisch R, Karin N, Steinman L, McDevitt HO (1994) A predominant role of integrin alpha 4 in the spontaneous development of autoimmune diabetes in nonobese diabetic mice. Proc Natl Acad Sci USA 91:12604-12608

21. Yang XD, Sytwu HK, McDevitt HO, Michie SA (1997) Involvement of beta 7 integrin and mucosal addressin cell adhesion molecule-1 (MAdCAM-1) in the development of diabetes in obese diabetic mice. Diabetes 46:1542-1547

22. Guttman JA, Li Y, Wickham ME, Deng W, Vogl AW, Finlay BB (2006) Attaching and effacing pathogen-induced tight junction disruption in vivo. Cell Microbiol 8:634-645

23. Verdaguer J, Schmidt D, Amrani A, Anderson B, Averill N, Santamaria P (1997) Spontaneous autoimmune diabetes in monoclonal $\mathrm{T}$ cell nonobese diabetic mice. J Exp Med 186:1663-1676

24. Gibson DL, Ma C, Bergstrom KS, Huang JT, Man C, Vallance BA (2008) MyD88 signalling plays a critical role in host defence by controlling pathogen burden and promoting epithelial cell homeostasis during Citrobacter rodentium-induced colitis. Cell Microbiol 10:618-631

25. Kim HS, Han MS, Chung KW et al (2007) Toll-like receptor 2 senses beta-cell death and contributes to the initiation of autoimmune diabetes. Immunity 27:321-333

26. Zhang Y, O'Brien B, Trudeau J, Tan R, Santamaria P, Dutz JP (2002) In situ beta cell death promotes priming of diabetogenic CD8 T lymphocytes. J Immunol 168:1466-1472

27. Luperchio SA, Schauer DB (2001) Molecular pathogenesis of Citrobacter rodentium and transmissible murine colonic hyperplasia. Microbes Infect 3:333-340

28. Anderson B, Park BJ, Verdaguer J, Amrani A, Santamaria P (1999) Prevalent CD8(+) T cell response against one peptide/ MHC complex in autoimmune diabetes. Proc Natl Acad Sci USA 96:9311-9316

29. Lieberman SM, Evans AM, Han B et al (2003) Identification of the beta cell antigen targeted by a prevalent population of pathogenic $\mathrm{CD}^{+} \mathrm{T}$ cells in autoimmune diabetes. Proc Natl Acad Sci USA 100:8384-8388

30. Flohe SB, Wasmuth HE, Kerad JB et al (2003) A wheat-based, diabetes-promoting diet induces a Th1-type cytokine bias in the gut of NOD mice. Cytokine 21:149-154

31. Maurano F, Mazzarella G, Luongo D et al (2005) Small intestinal enteropathy in non-obese diabetic mice fed a diet containing wheat. Diabetologia 48:931-937

32. Funda DP, Kaas A, Tlaskalova-Hogenova H, Buschard K (2008) Gluten-free but also gluten-enriched (gluten+) diet prevent diabetes in NOD mice; the gluten enigma in type 1 diabetes. Diabetes Metab Res Rev 24:59-63

33. Richer MJ, Horwitz MS (2009) Coxsackievirus infection as an environmental factor in the etiology of type 1 diabetes. Autoimmun Rev 8:611-615

34. van der Werf N, Kroese FG, Rozing J, Hillebrands JL (2007) Viral infections as potential triggers of type 1 diabetes. Diabetes Metab Res Rev 23:169-183 
35. Vaarala O (2008) Leaking gut in type 1 diabetes. Curr Opin Gastroenterol 24:701-706

36. Oikarinen M, Tauriainen S, Honkanen T et al (2008) Detection of enteroviruses in the intestine of type 1 diabetic patients. Clin Exp Immunol 151:71-75

37. Wen L, Ley RE, Volchkov PY et al (2008) Innate immunity and intestinal microbiota in the development of type 1 diabetes. Nature 455:1109-1113

38. Raine T, Zaccone P, Mastroeni P, Cooke A (2006) Salmonella typhimurium infection in nonobese diabetic mice generates immunomodulatory dendritic cells able to prevent type 1 diabetes. J Immunol 177:2224-2233
39. Hall JA, Bouladoux N, Sun CM et al (2008) Commensal DNA limits regulatory $\mathrm{T}$ cell conversion and is a natural adjuvant of intestinal immune responses. Immunity 29:637-649

40. Rakoff-Nahoum S, Paglino J, Eslami-Varzaneh F, Edberg S, Medzhitov R (2004) Recognition of commensal microflora by tolllike receptors is required for intestinal homeostasis. Cell 118:229-241

41. Slack E, Hapfelmeier S, Stecher B et al (2009) Innate and adaptive immunity cooperate flexibly to maintain host-microbiota mutualism. Science 325:617-620

42. Barbara G, Stanghellini V, Brandi G et al (2005) Interactions between commensal bacteria and gut sensorimotor function in health and disease. Am J Gastroenterol 100:2560-2568 